\title{
Plataforma Mlearning como apoyo a las actividades de enseñanza aprendizaje en la física mecánica
}

\section{Mlearning platform to support learning activities in physical education mechanics}

\author{
Ing. Fabián José Ramos Torres ${ }^{1}$ \\ ${ }^{1}$ Magíster en Software Libre, Especialista en Auditoría de Sistemas, Profesor Tiempo Completo \\ Universidad Autónoma del Caribe, Grupo de Investigación SINT, framos@uac.edu.co
}

Recibido 17/08/11, Aceptado 05/09/2012

\section{RESUMEN}

El desarrollo de las tecnologías de la información y las comunicaciones ha generado la incorporación de nuevas estrategias educativas en busca de un aprendizaje significativo. Esto, junto con el crecimiento de las redes inalámbricas y móviles, así como el aumento en el uso de dispositivos móviles impone un nuevo desafío para su aprovechamiento en el marco de la Tics con el fin de ampliar los servicios educativos digitales, haciendo énfasis en las actividades académicas virtuales que permitan la búsqueda de contenidos, la evaluación temática y el debate. Este proyecto dirige sus esfuerzos hacia el diseño e implementación de una plataforma móvil como apoyo al proceso de enseñanza aprendizaje en los cursos de física mecánica de los programas de ingeniería en las instituciones de educación superior. Conformada por un entorno web, un conjunto de objetos virtuales de aprendizajes animados y una aplicación móvil bajo tecnología Java ME que permita a los estudiantes el acceso a la información en cualquier momento y desde cualquier lugar.

Palabras clave: Física mecánica, Java Me, M-learning, Tics, móviles.

\begin{abstract}
The development of information technology and communications have resulted in the incorporation of new strategies in search of meaningful learning. This, together with the growth of wireless and mobile networks and the increased use of mobile devices imposes a new challenge for its use in the context of ICTs to expand digital educational services, with emphasis on virtual academic activities to enable content search, evaluation and discussion topics. This project directs its efforts towards the design and implementation of a mobile platform to support the teaching-learning process in physics courses mechanical engineering programs in institutions of higher education. Formed by a web environment, a set of virtual objects and animated learning in a mobile application Java ME technology that allows students access to information anytime, anywhere.
\end{abstract}

Keywords: Mechanical physics, Java ME, m-learning, free software, mobiles.

\section{INTRODUCCIÓN}

El proceso de enseñanza y la generación de competencias en las ciencias básicas de las Facultades de Ingeniería es uno de los factores claves para el éxito de los estudiantes de los primeros semestres de su carrera, además de ser piedra angular en su formación profesional. No es de ocultar los problemas que se presentan tanto en el estudio y asimilación de los contenidos de las mismas como la metodología de enseñanza de los profesores, viéndose esto reflejado en los resultados de las evaluaciones y como consecuencia de esto el incremento de la deserción estudiantil temprana Lo primero algo inherente a la complejidad de las ciencias exactas y lo segundo debido a la falta de aplicación de nuevas estrategias pedagógicas y tecnológicas que acompañen a los estudiantes en su tiempo de estudio independiente.

Pero gracias al avance de las Tics en la educación se han creado nuevas oportunidades y alternativas para alimen- 
tar este camino, viéndose el surgimiento de plataformas o portales educativos que permiten a la comunidad académica de crear espacios para el intercambio de conocimiento y cultura, siendo el Internet y las autopistas de comunicaciones el agente de cambio.

Los nuevos cambios tecnológicos influyen de forma determinante en la educación, generando un flujo constante en la aparición de herramientas educativas para el mundo académico, ahora nos encontramos en la era de las redes inalámbricas y móviles, información al instante en cualquier lugar y tiempo, al alcance de nuestras manos. El uso de dispositivos móviles (celulares, agendas de mano, iphone entre otros) se incrementa en la población mundial de forma inimaginable, y que pueden en un futuro muy cercano desplazar a los computadores personales del reinado informático, teniendo en cuenta el poder en procesamiento y almacenamiento que tendrán gracias al avance de ciencias como la nanotecnología y los microsistemas.

\section{TEORÍA}

\subsection{E-learning y M-learning}

Desmond Keegan define el E-learning como el arte de la educación a distancia en el tiempo de la escritura [1], es decir una forma de educación a distancia apoyada con las Tics. El E-learning crea ambientes de aprendizaje centrados en el estudiante utilizando la tecnología informática.

Los sistemas E-learning deben considerar ocho aspectos: el diseño instruccional, pedagógico, tecnológico, de interfaz, evaluación, gerencia, soporte y ética de uso [2].

El E-learning se puede clasificar según los medios tecnológicos que utilice en CBT (Computer based training) o CAI (Computer assisted instruction) basado en la lectura y en mecanismos pregunta respuesta; IBT (Internet based training) extiende el CBT por medio del uso de internet para la divulgación de contenidos y actividades; y WBT (web based training) aprendizaje hacienda uso de la Web.

El éxito del E-learning ha estado soportado por la penetración del internet en todos los países del mundo, en todos los estratos y en diferentes idiomas. El uso de internet en el mundo ha crecido en un 564.4\% desde el año 2000, pasando de 361 millones a más de 2.400 millones de usuarios, logrando América Latina el tercer mayor incremento con el $1310.8 \%$ después de África y el Medio Este, pasando de 18 millones a más de 250 millones en el 2012, teniendo el $42,9 \%$ de penetración de una población estimada en 593 millones. Colombia ocupa en Suramérica el segundo lugar con una participación regional del $11.2 \%$, superada por sólo por Brasil [3]. En Colombia la cantidad de usuarios en internet supera los 25 millones, alcanzando una penetración del 55,9\% con un crecimiento del $2747 \%$ en la última década, y un total de suscriptores a internet de casi tres millones repartidos en acceso de banda ancha, internet móvil y telefonía de marcación. Estas cifras responden a los esfuerzos del gobierno nacional a través de su proyecto de Agenda de Conectividad establecida en el año 2000 y que ha sido mantenida por los subsiguientes gobiernos nacionales.

A pesar del éxito del E-learning y su alto grado de madurez alcanzado, el desarrollo de los sistemas de comunicaciones móviles ha sido el gran detonante de una nueva forma de interacción entre aprendices y tutores, el M-learning, el cual lo define Alexander Dye como "una clase de aprendizaje que puede ocurrir en cualquier lugar y en cualquier momento con la ayuda de un dispositivo móvil capaz de presentar contenido y suministrar conexión inalámbrica para la comunicación entre estudiantes y profesores a través de un entorno administrativo educacional" [4].

En el M-learning se identifican claramente las siguientes características y ventajas del M-learning [5]:

- Movilidad: desde un punto vista tecnológico se refiere a poder trasladarse en diferentes entornos geográficos sin perder la capacidad de estar conectado a las plataformas de comunicaciones; y desde el punto de vista educativo la capacidad de aprender desde diferentes contextos y comunicar las experiencias en diferentes entornos.

- Ubicuidad: que permite que los actores del proceso educativo siempre estén en línea, conectados con el mundo en cualquier instante y desde cualquier lugar.

- Oportuno: elimina las restricciones de espacio y tiempo, su tecnología siempre está ahí para ser utilizada, está en el momento y en lugar indicado, en el mundo de hoy siempre tenemos un dispositivo móvil a la mano.

- Personalizado: tiene la capacidad de entregar servicios teniendo en cuenta las necesidades de los estudiantes para su auto aprendizaje.

- Tiempo real: el conocimiento que queremos lo podemos obtener inmediatamente, en el instante en que los hechos estén sucediendo.

- Interactivo: hay un canal de comunicación bidireccional entre estudiantes y profesores permitiendo la colaboración y la acción dialógica.

- Virtualización: se crean escenarios que representan la realidad y con los cuáles profesores y estudiantes interactúan dinámicamente. 
- Digitalización: la existencia de recursos digitales multimediales, de una red de comunicaciones que soporta un flujo de paquetes de bits y terminales móviles que guardan información.

- Flexibilidad: los dispositivos móviles ofrecen diferentes aplicaciones y servicios como, reproductores de música y vídeo, conexiones a redes inalámbricas Wi$\mathrm{Fi}$, cámaras fotográficas y de vídeo integradas, juegos interactivos, internet móvil, organizadores, agendas, tareas, mensajes de texto y multimedia, internet móvil entre otros, que difieren de acuerdo a la tecnología del dispositivo.

- Colaboración: los grupos de estudio se apoyan y comparten conocimientos para la ejecución de trabajos, solución de inquietudes y búsqueda de información.

- Portabilidad: las aplicaciones pueden ejecutarse en variedad de dispositivos y sistemas operativos con poco o ningún esfuerzo de configuración.

- Motivación: esta nueva forma de enseñanza y aprendizaje genera gran interés por los usuarios, especialmente a los estudiantes por ser la tecnología parte inseparable de su forma de vida.

El panorama mundial es ideal para la convivencia de la educación móvil, según las estadísticas del ITU World Telecomunication el total de suscriptores en telefonía móvil a nivel mundial alcanzó la cifra de 6.000 millones a finales del 2011, habiendo 86 suscriptores por cada cien habitantes, cifra que supera en más de cinco veces la de 15 suscriptores por cada cien en el año 2001 [6].

Según la organización 3gAméricas América Latina es el tercer mercado más grande de telefonía móvil, en volumen, a nivel mundial después de Asia y África, con más de 630 millones de conexiones hasta el cuarto trimestre de 2011, que representa el 10\% mundial y se espera que la región cuente con setecientos cincuenta mil millones de conexiones para el año 2015. La banda ancha móvil ha superado a la banda ancha fija como primer opción para acceder a Internet en América Latina. Las suscripciones de banda ancha móvil han aumentado un $127 \%$ por año durante los últimos cinco años y se prevé que continúen creciendo a un $50 \%$ por año durante los próximos cinco años. La cobertura de los servicios $2 \mathrm{G}$ y $3 \mathrm{G}$ en Colombia es de 94 y el 52\% respectivamente [7]. La industria móvil contribuye activamente para mejorar y difundir la educación en Latinoamérica, tanto al posibilitar el aprendizaje a través de dispositivos móviles como favoreciendo el acceso a Internet y móvil en las escuelas, por ejemplo Nokia y Telefónica están ofreciendo contenidos educativos a escuelas remotas a través de la tecnología móvil en toda la región por medio del programa EducaRed. En Colombia, Tigo ha donado salas de informática totalmente equipadas y ha prestado servicios de Internet a instituciones educativas en zonas de bajo nivel económico, beneficiando así a más de 3.000 niños.

\subsection{Dispositivos y software móvil}

Los dispositivos móviles usados en el M-learning pueden ser categorizados en dos tipos principales, los teléfonos móviles y los computadores móviles. Entre los primeros se encuentran teléfonos móviles y teléfonos inteligentes y a los segundos pertenecen los organizadores, PDA's, Tablet PC's, Pen Tablet, WebPAD, PaceBook, MiniPC, SmartBook, Sub NoteBook, Mini NoteBook, laptop, Smartboard y CarKit entre otros. Además existen diferentes accesorios tales como interfaces cognitivas, grabador-receptor digital, bolígrafos digitales, impresoras portátiles, juegos, pantallas, teclados, Parlantes HiFi, Ratón, Impresora y DigiCam por mencionar algunos [8].

En Colombia el mercado de los teléfonos móviles en el año 2009 Nokia lidera con su gama de productos alcanzando el $36.4 \%$ del mercado, seguida de Samsung con el 19.5\%, LG con el $10.1 \%$, Motorola con el $4.8 \%$ y Sony Ericsson con el 4.5. En Colombia para finales del 2008 existían más de 41 millones de dispositivos móviles activados, y entre los años 1998 y 2007 la densidad celular pasó del 4.4\% al $85.45 \%$ [9].

Al hablar de software móvil nos referimos a los sistemas operativos, lenguajes de desarrollo, entornos de desarrollo, bases de datos, utilidades de software y equipos móviles que conforman un sistema sinérgico para la implementación de soluciones educativas basadas en M-learning.

Entre los sistemas operativos de actualidad se encuentran:

- Symbian: sistema operativo principal de la familia Nokia de 32 bits, multitarea, soporta GSM, 3G, email, sincronización de datos.

- Android: es una pila de software para dispositivos móviles que incluye un sistema operativo basado en Linux, middleware y aplicaciones clave. El SDK de Android proporciona las herramientas y las API necesarias para empezar a desarrollar aplicaciones en la plataforma Android usando el lenguaje de programación Java.

- $\quad$ Blackberry OS: sistema operativo que se ejecuta sobre computadoras de mano, desarrollado por RIM (Research In Motion) y cuyo núcleo está basado en $\mathrm{C}++$. Incorpora funcionalidades de envío de mensajes de texto, correo electrónico y mensajería instantánea [10]. 
- Palm OS: sistema operativo que corre en los smartphones y PDA's, competencia del Windows Mobile, desarrollado por PalmSource Inc. Posee aplicaciones usuales para los usuarios de teléfonos móviles incluyendo además software de reconocimiento de voz llamado Graffiti, identificación biométrica y soporte para tarjetas inteligentes [11].

- iPhone OS: es un sistema operativo de Apple para dispositivos móviles basado en Mac Os X, caracterizado por su eficiencia y larga vida de su batería, permite la multitarea, integración completa con el hardware. El iPhone 4.0 posee aceleración gráfica, pantalla Retina, GPS, Wi-fi y de uno a dos micrófonos [12].

A junio de 2010 los principales sistemas operativos estaban liderados por Symbian OS con el 33.39\%, iPhone OS con el $26.66 \%$, RIM con el $14.98 \%$, Sony Erickson con el $5.79 \%$, Android con el 3.95\%, Samsum con el 3.34 y otros con el $9.81 \%$ [13]. El de mayor crecimiento en el período junio09-junio10 es el RIM que pasó del 7.91\% al 16.62\%. En cuanto a su participación en el mercado a febrero del 2010 dominaba Symbian OS con el 47\%, Blackberry con casi el $20 \%$, iPhone con el 14\%, Windows Mobile con el 8\%, Linux con el 5\%, Android con el 4\% y Web OS con el 2\% [14]. Para el 2012 se espera que el sistema operativo Android supera al iPhone llegando al 14.5\% según estimaciones de Gartner.

Para el desarrollo de las aplicaciones móviles existen una serie de plataforma y lenguajes destinados para tales fines. Como iniciativa de Microsoft aparece el .NET Compact FrameWork que soporta desarrollos en Visual Basic .NET y C\# y que se ejecuta sobre Windows Mobile; Sun Microsystems presenta su plataforma J2ME también denominada JavaME que sigue los pasos la J2SE y la J2EE pero ahora para dispositivos móviles, contando con su máquina virtual llamada KVM; como otra alternativa de desarrollo se encuentra BREW, desarrollada por Qualcomm, de gran uso en los USA y Asia, teniendo como lenguaje nativo $\mathrm{C} / \mathrm{C}++$. También se pueden desarrollar en lenguajes como Perl para Series 60 y Python.

Referente a entornos integrados de desarrollo podemos mencionar a Netbeans con su paquete Mobility y JavaFx Mobile, Eclipse, UIQ para Symbian, JBuilder y BlueJ. Flash Lite, K-Toon y Synfig para desarrollo de animaciones para móviles.

\subsection{Metodología del proyecto}

\section{- FASE DE IDENTIFICACIÓN}

En esta fase de analizó la situación actual del uso de las Tics en la asignatura de Física Mecánica realizando una encuesta a estudiantes y entrevistas a docentes indagando principalmente por las tecnologías Web y Móvil en la institución. Se determinó que el uso de sistemas LMS en la institución se ha consolidado a través de la implementación de la herramienta Moodle, cuyo uso se ha hecho obligatorio dentro de las estrategias de seguimiento y evaluación del trabajo independiente. Según los resultados de la encuestas se pudo concluir

- Encuesta a estudiantes relacionado con el uso de Tics en la asignatura de Física teniendo en cuenta el tamaño de la muestra calculado

- Análisis de los resultados de la encuesta y revisión de las plataformas Tics de la institución para la descripción de la situación actual.

- Selección de profesores basado en sus aptitudes en relación a las Tics que nos apoyaran en el diseño funcional de los contenidos, simulaciones y evaluaciones del curso.

- Desarrollo de la aplicación Web y Móvil en el lenguaje Java utilizando las plataformas J2EE y Java ME.

- Montaje y configuración del servidor Linux y el Apache Tomcat.

- Selección de los dispositivos móviles para la instalación de la aplicación móvil

- Verificación del acceso desde equipos cliente a la aplicación Web.

- Evaluación del acceso desde equipos clientes móviles al repositorio de contenidos y objetos de aprendizaje.

- Pruebas de acceso y uso de la aplicación móvil.

- Realizar pruebas de funcionalidad a la aplicación móvil.

- Toma de tiempos de respuestas en descarga de archivos en los clientes móviles.

La encuesta realizada a una muestra de estudiantes de primer semestre de ingeniería en los cursos de Física I, indicó que el $98 \%$ posee teléfono celular y que solamente el $27,4 \%$ dice haber utilizado el móvil como una herramienta de aprendizaje; también el $98 \%$ indica que el profesor del curso no ha utilizado un dispositivo móvil como herramienta de comunicación y de enseñanza con sus estudiantes y el $84.3 \%$ le gustaría tener una aplicación móvil educativa como apoyo para la enseñanza de la Física.

Realizando un análisis del rendimiento de los estudiante en el área de la Física se identificó que el promedio de las calificaciones de los estudiantes son inferiores al 3.6, un promedio regular, del cual podemos deducir muchos factores como la baja formación en el colegio, fallas en la selección de la carrera profesional, problemas personales y familiares, problemas de adaptación al ambiente universitaria, desmotivación hacia las formas de enseñanzas, responsabilidades laborales, viajes permanentes por motivos laborales, etc.; pero ante los cuales debemos actuar para 
evitar el aumento de la deserción y de la frustración estudiantil. Para esto debemos crear herramientas que faciliten y disminuyan esta problemática permitiendo que desde cualquier lugar y espacio se puedan acceder a recursos educativos y responder actividades en línea.

Para el desarrollo de la aplicación móvil se trabajó con un grupo de profesores que imparten el curso de Física I (Física Mecánica) en la institución para hacer el diseño de las simulaciones, la selección y diseño de los contenidos, evaluaciones, cuestionarios, fotos y videos que servirán de recursos para los estudiantes. Se consideró como característica principal para la selección de los profesores aquellos que posean habilidades en el uso de las Tics, para esto se identificarán los docentes que han recibido capacitación en el uso de la plataforma e-learning de la institución y hayan realizado capacitación en Tics.

La aplicación móvil se instaló en tres (3) equipos móviles basados en sistema operativo Symbian OS, ver tabla 1, y se evaluó la funcionalidad y eficacia de la aplicación móvil en los aspectos de: calidad de la interfaz gráfica, rapidez de descarga de los contenidos de los cursos, desarrollo de las evaluaciones y cuestionarios, administración de perfiles y roles, calidad de audio y pertinencia de las simulaciones.

Las pruebas de acceso desde el dispositivo móvil fueron eficaces pero su eficiencia. Las pruebas de usuario final y retroalimentación se efectuarán con una muestra de estudiantes de primer semestre del 2011-02 en los cursos de Física I.

Tabla 1. Descripción dispositivos móviles usados para las pruebas (tomado de Nokia.com)

Table 1. Description used mobile devices for testing (taken form Nokia.com).

\begin{tabular}{|c|c|c|c|c|}
\hline Dispositivo & Resolución & Teclas y método de entrada & Aplicaciones & Red de datos \\
\hline $\begin{array}{l}\text { Nokia } 5130 \\
\text { ExpressMusic }\end{array}$ & $\begin{array}{l}320 \times 240 \text { píxeles } \\
\text { (QVGA). Hasta } \\
\text { 256k colores }\end{array}$ & $\begin{array}{l}\text { Teclado numérico } \\
\text { Teclas dedicadas de música y de } \\
\text { volumen } \\
\text { Comandos de voz }\end{array}$ & $\begin{array}{l}\text { Java }{ }^{\mathrm{TM}} \\
\text { MIDP } 2.0 \\
\text { Flash Lite } 2.0\end{array}$ & $\begin{array}{l}\text { CSD,GPRS release } 4 \text {, clase B con } \\
\text { GPRS multislot clase } 32 \\
\text { EDGE GPRS multislot clase } 32 \\
\text { Soporte para TCP /IP } \\
\text { Capacidad para funcionar como } \\
\text { módem de datos } \\
\text { Soporte para sincronización de } \\
\text { contactos, agenda y notas del MS } \\
\text { Outlook }\end{array}$ \\
\hline $\begin{array}{l}\text { Nokia } 5130 \\
\text { ExpressMusic }\end{array}$ & $\begin{array}{l}\text { QVGA de } 2.0 " \text { ", } \\
320 \times 240 \text { píxeles } \\
\text { con hasta } 16 \text { mi- } \\
\text { llones de colores }\end{array}$ & $\begin{array}{l}\text { Teclas dedicadas para música y } \\
\text { juegos N-Gage. Tecla Navi }{ }^{\mathrm{TM}} \text { en } 8 \\
\text { direcciones, optimizada para jue- } \\
\text { gos }\end{array}$ & $\begin{array}{l}\text { MIDP Java } \\
2.1 \\
\text { Macromedia } \\
\text { Flash Lite } 3.0\end{array}$ & $\begin{array}{l}\text { HSDPA categoría } 6 \text { (hasta } 3.6 \\
\text { Mbits/s) } \\
\text { GPRS: Clase B, multislot clase } 32 \\
\text { EGPRS: Multislot clase } 32\end{array}$ \\
\hline $\begin{array}{l}\text { Nokia } 5800 \\
\text { XpressMusic }\end{array}$ & $\begin{array}{l}\text { Resolución } 640 \text { x } \\
360 \text { píxeles } \\
\text { Hasta } 16 \text { millones } \\
\text { de colores }\end{array}$ & $\begin{array}{l}\text { Soporte para control con lápiz, plec- } \\
\text { tro y toque táctil para entrada de } \\
\text { texto e interfaz de usuario (teclado } \\
\text { alfanumérico, teclado QWERTY } \\
\text { mini y completo, reconocimiento } \\
\text { de escrita manual, escrita manual } \\
\text { en pantalla completa para chino). } \\
\text { \# Tecla de toque dedicada a la Ba- } \\
\text { rra de media para acceso a música, } \\
\text { galería, compartir en línea }\end{array}$ & $\begin{array}{l}\text { Java TM } \\
\text { MIDP } 2.0 \\
\text { Flash Lite } 3.0\end{array}$ & $\begin{array}{l}\text { CSD } \\
\text { HSCSD } \\
\text { GPRS/EDGE clase B, multislot clase 32, } \\
\text { velocidad total } 296 / 177 \text { kbps (DL/UL) } \\
\text { WCDMA } 850 / 1900 \text { con paquete de da- } \\
\text { tos y voz simultáneos, velocidad total } \\
\text { PS de } 384 / 384 \mathrm{kbps} \text { (UL/DL) } \\
\text { CS velocidad total de } 64 \mathrm{kpbs} \\
\text { HSDPA cat. 6, velocidad total de } 3,6 \\
\text { Mbps (DL) } \\
\text { WLAN IEEE } 802.11 \mathrm{~b} / \mathrm{g} \text { (apenas versión } \\
\text { 3G) } \\
\text { Soporte para TCP/IP }\end{array}$ \\
\hline
\end{tabular}

El sistema está complementado con un módulo Web instalado en un alojamiento basado en servidor Apache y base de datos MySql, que permite la configuración de usuarios, cursos, contenidos y evaluaciones del sistema. Los docentes accederán al aplicativo Web desde computadores personales en sus domicilios o en los equipos de de la institución. Las pruebas de funcionalidad a nivel local mostraron un comportamiento óptimo desde los navegadores Internet Explorer y Mozilla FireFox los cuales son los utilizados en la institución.

\section{RESULTADOS}

El proyecto deja como resultados la aplicación móvil, la aplicación web, animaciones Flas Lite, audios y textos de contenidos de la física mecánica. Las pruebas de funcionalidad fueron exitosas teniendo en cuenta el cumplimiento de los requisitos definidos, pero la eficiencia de las conexiones y descargas de archivos superaron el tiempo indicado, lo cual indica la necesidad de realizar diseños que 
permitan mantener contenido tipo offline y de tamaños de archivos adecuados para una descarga más rápida.

El sistema fue instalado en dispositivos celulares de estudiantes que soportarán las especificaciones CLDC 1.0/1.1 y MIDP 2.0, los cuáles accedieron a sus cuentas de usuario creadas previamente logrando.

\subsection{Plataforma Mlearning}

El objetivo principal de la plataforma es permitir la realización de actividades de enseñanza aprendizaje entre docentes y estudiantes desde dispositivos móviles, como apoyo al desarrollo de competencias en el curso de física mecánica de la Facultad de Ingeniería de la Universidad Autónoma del Caribe.

El sistema implementa como principales funcionalidades:

- Actualizar información de perfil

- Inscripción de cursos por parte del estudiante

- Descargar recursos de audio, animaciones, videos.

- Lectura de textos

- Realizar evaluaciones on-line

- Ver mensajes

- $\quad$ Ver novedades

- $\quad$ Ver actividades

La aplicación está estructurada una aplicación Web, una aplicación móvil y un repositorio de objetos de aprendizaje.

\subsection{Aplicación Web}

Basada en servlets, archivos jsp y Ajax, ver Figura 1, y organizada en tres (3) paquetes: beans, modelo y servlets. El paquete beans contiene las clases relacionadas con la lógica del negocio. El paquete model utilizado básicamente para el manejo de las colecciones correspondientes a las consultas a la base de datos. El paquete servlets gestiona las peticiones del cliente Web.

Figura 1. Interfaz Principal Aplicación Web.

Figure 1. Principal Web Application Interface.

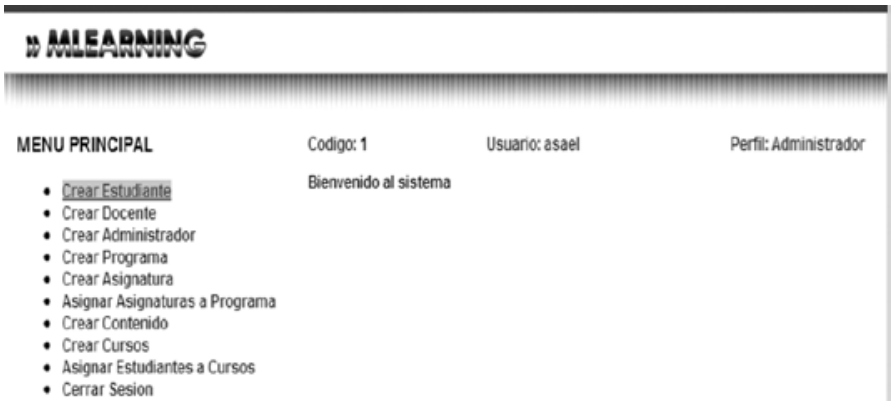

\subsection{Aplicación móvil}

La aplicación móvil está orientada básicamente a los estudiantes para que accedan a los recursos y actividades montadas por el profesor, ver Figura 2. La solución móvil está dividida en tres paquetes de clases, el paquete co.edu. mlearning.model donde se encuentran las clases de la lógica del negocio del modelo; el paquete co.edu.mlearning. resources el cual guardan las imágenes que se utilizan dentro del proyecto; y el paquete co.edu.mlearning.view que agrupa las clases de la interfaz y lógica de la aplicación.

Como complemento se crearon los servlets que gestionan las peticiones del móvil y que se encuentran alojados en el paquete servlet de la solución. Se emplea la configuración CLDC 1.1 y el perfil MIDP 2.1 lo cual implica que los dispositivos móviles que se utilicen deben poseer estás características para que la aplicación se pueda instalar y ejecutar adecuadamente.

Para el acceso al servidor se utilizaron las clases del paquete GFC (Generic Connection Framework) Connector y HttpConnection y las clases InputStream y OutStream del paquete io. Se definió la variable Http en el JAD (Java Application Descriptor) para colocar allí la ruta del servidor http al momento de crear el ejecutable. Esta variable es leída y asignada a una variable estática del proyecto. Todos los menús de consulta son generados teniendo en cuenta la información que se encuentra en la base de datos.

Se desarrollaron clases utilitarias para el proyecto como, la clase Split para la extracción de caracteres; la clase Downloader para la descarga de archivos y la clase LeerXml para la extracción de formatos XML.

Figura 2. Interfaz Principal Aplicación Móvil. Figure 2. Principal Mobile Application Interface.

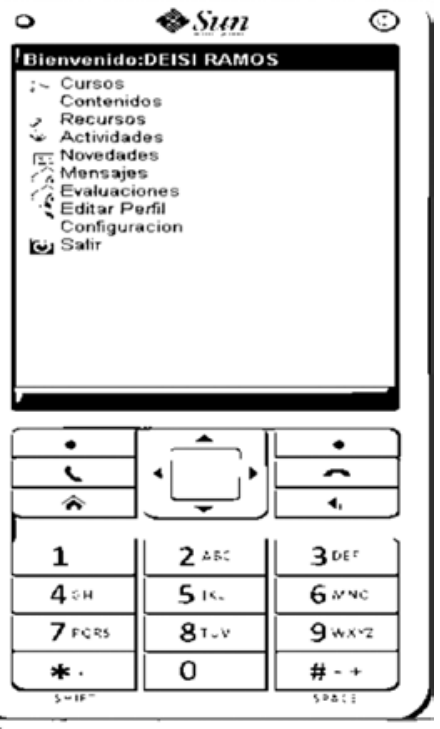




\subsection{Recursos virtuales de aprendizaje}

Los recursos virtuales de aprendizaje fueron diseñados teniendo en cuenta las temáticas que se imparten en el curso de Física I en los que se encuentran:

Archivos de texto: archivos en formato texto (txt), para conveniencia de los docentes, los cuales contendrán las definiciones, conceptos teóricos y explicaciones de cada uno de los temas. Serán leídos y visualizados en el dispositivo móvil del estudiante cuando este lo requiera, siendo habilitados para su acceso cuando este lo estipule conveniente. Estos archivos pueden ser creados con el bloc de notas o cualquier editor similar y se aconseja que contenga los siguientes ítems: título, tema(s), cuerpo, bibliografía (ver Anexo 3). Estos archivos reposaran en el servidor web en una carpeta de recursos denominada Textos de la aplicación Web.

Archivos de Audio: estos archivos serán creados por los docentes en herramientas de grabación y serán incorporado a la plataforma. Cada tema del curso de física mecánica podrá incorporar estos recursos en el repositorio de contenidos y serán accedidos por los estudiantes desde su dispositivo móvil haciendo peticiones al servidor Web. Deben tener una extensión wav y su tamaño y se recomienda que su tamaño no sea superior a $1 \mathrm{MB}$. Los docentes pueden realizar la grabación con la herramienta Grabadora de Sonidos de Windows $u$ otra herramienta, para disminuir el tamaño de los mismos si es superior a $1 \mathrm{MB}$, editarlos y transformarlos con las siguientes características: Baudio Rate 6000, Bit de profundidad de 8 y canal Mono. Estos archivos reposaran en el servidor web en una carpeta de recursos denominada Audio de la aplicación Web.

Animaciones: estos archivos brindarán al estudiante la posibilidad de ver, escuchar e interactuar con los objetos de la misma, buscando mayor interés y motivación en el estudio de los temas de la física mecánica. Cada tema del curso de física mecánica podrá incorporar estos recursos en el repositorio de contenidos y serán accedidos por los estudiantes desde su dispositivo móvil haciendo peticiones al servidor Web. Estos archivos reposaran en el servidor web en una carpeta de recursos denominada Animaciones de la aplicación Web.

Se desarrollaron doce (12) animaciones que explican conceptos de la física en las temáticas de vectores, magnitudes, leyes de Newton, cinemática, trabajo y energía, ver ejemplos Figuras 3 y 4 .
Figura 3. Vista Animación M.C.U

Figure 3. View Animation M.C.U.

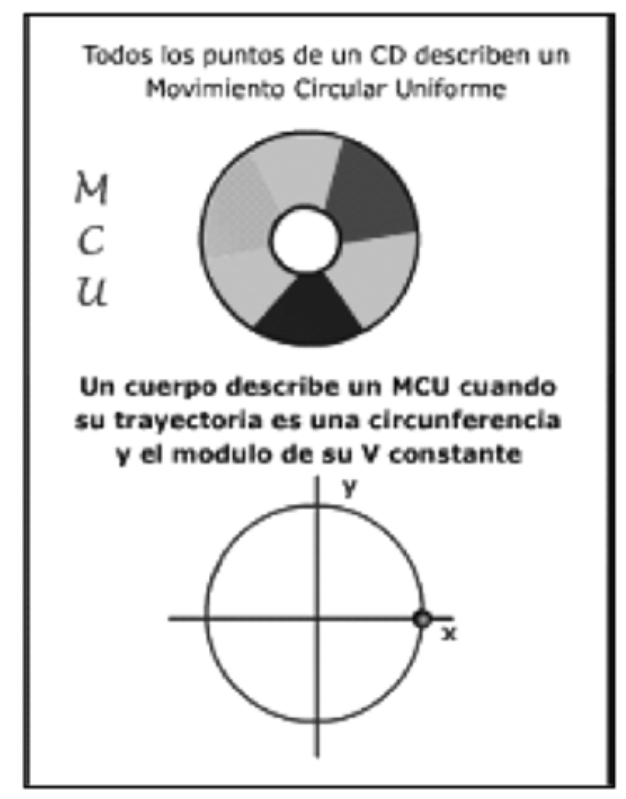

Figura 4. Vista Animación Leyes de Newton, Figure 4. View Animation's Laws Newton

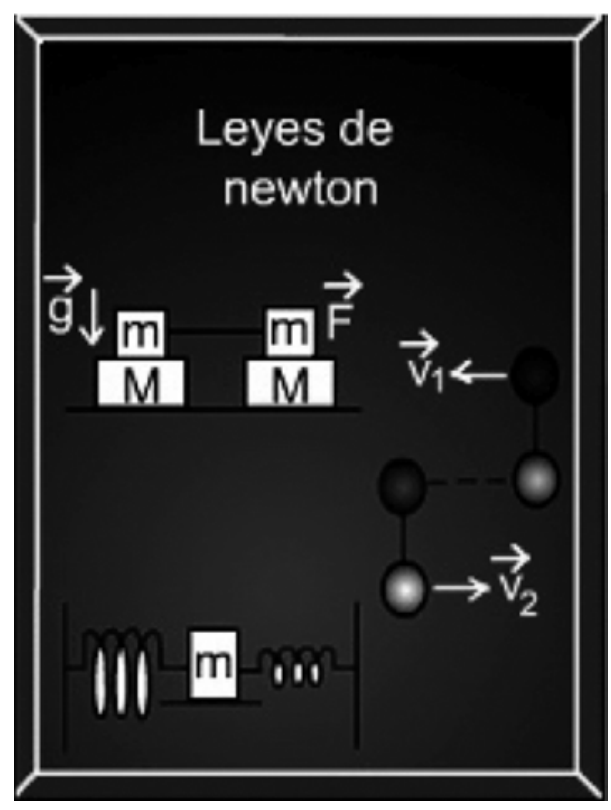

Evaluaciones: basadas específicamente en los tipos de cuestionarios con opción múltiple y falso/verdadero en formato $\mathrm{Xml}$.

\section{CONCLUSIONES}

El proyecto permitió identificar los siguientes aspectos importantes en el desarrollo de soluciones m-learning: 
- Las comunicaciones móviles es una nueva y excelente alternativa de apoyo para los docentes y estudiantes dentro de su quehacer educativo.

- El m-learning no sólo contempla el contexto tecnológico sino también su aspecto organizacional, curricular, de formación de docentes y estudiantes y el desarrollo de recursos de aprendizajes virtuales propios.

- Las soluciones m-learning deben tener básicamente tres estructuras primordiales, los recursos virtuales de aprendizaje, que en esta investigación corresponde a recursos de texto, de audio y de animaciones, los cuales deben ser elaborados por los docentes para la pertinencia de los mismos. La aplicación Web debe permitir el registro de la información de los actores educativos, los recursos y actividades; y la aplicación móvil desde donde se puede acceder a los mismos y permitir reproducir los diversos recursos.

- Las animaciones deben ser realizadas con herramientas de diseño adecuadas y con la participación activa del docente.

- Los dispositivos móviles deben tener las características adecuadas para la funcionalidad de la aplicación, para aplicaciones java estos deben contar configuración CLDC 1.0/1.1 y MIDP 2.0 ó superior.

- Es necesario implementar operaciones offline en la aplicación móvil utilizando almacenamiento persistente y colecciones de datos para guardar la información en el dispositivo, disminuyendo la cantidad de accesos a la red, definiendo espacios de sincronización de datos.

- Desarrollar funcionalidades en la aplicación móvil para que el docente pueda realizar acciones que solamente brinda la aplicación Web.

- Las evaluaciones en los dispositivo móvil deben seguir formatos internacionales (IMS, SCORM) que permitan ser utilizados en diferentes plataformas LMS.

- Es indispensable la conformación grupos de diseño para la actualización y la creación de nuevas animaciones.

- Es prioritario crear otros tipos de actividades como foros, glosarios, diversas modalidades de cuestionarios, descarga de videos (flv, wma), entre otros.

- Desarrollar soluciones móviles que trabajen en conjunción con LMS existentes como aplicaciones Web es una excelente alternativa que nos permite centrarnos en la ambiente móvil y aprovechar las opciones que estas nos brindan.

\section{REFERENCIAS}

[1] Keegan, Desmond., The future of learning: From eLearning to M-Learning, FernUniversität - Hagen, Germany, 2002.
[2] Boneu, Josep M, Plataformas abiertas de E-learning para el soporte de contenidos educativos abiertos, Revista de Universidad y Sociedad del Conocimiento (RUSC), 4 (1), 35-46, 2007.

[3] Internet World Stats, Usage and population statistics [Internet]. Disponible desde: [acceso 2010, 28 de Junio].

[4] Dye, Alexander, 2001, Mobile Education-A Glance at The Future [Internet], Bekkestua - Norway. Disponible desde: < http://www.dye.no/articles/a_glance_at_the_future >, [Acceso 10 de mayo de 2010].

[5] 2009 International Conference on Wireless Networks and Information Systems, 2. 2009. Research of One Mobile Learning System, Yi, Jin. Kathmandu, Nepal. IEEE Computer Society.

[6] ITU. Medida de la sociedad de la información, 1-3, 2012.

[7] GSMA. Observatorio Móvil de América Latina, 10-13, 2011.

[8] EUROCON The International Conference on "Computer as a Tool", 2007. An Alternative to the Traditional Methods in Education - M-Learning: a Glance into the Future. Marghescu, G., Hicioreanu, Teodora., y Marghes$\mathrm{cu}$, Ion.Warsaw. EUROCON.

[9] Pettey, Christy. Gartner [Internet]. Egham, UK. Disponible desde: http://www.gartner.com/newsroom/id/ 1306513 [acceso 2010, 8 de Julio].

[10] Shelly, Gary., y Vermmat, Misty., Discovering computers 2009 brief, Course Technology, Cengage Learning, Boston-USA, 2008.

[11] StatCounter GlobalStats [Internet]. Disponible desde: http://gs.statcounter.com/\#mobile_os-ww-monthly200906-201007 [acceso 2010, 3 de Julio].

[12] Apple.com [Internet]. Disponible desde: http:/ / www. apple.com/iphone/ios4/ [acceso 2010, 9 de Julio].

[13]Android Developers [Internet]. Disponible desde: http:/ / developer.android.com/guide/basics/what-isandroid.html [acceso 2010, 3 de Julio].

[14] Cell Phones Operating Systems [Internet]. Disponible desde: http:/ / cellphones.org/blog/cell-phone-os/ [acceso 2010,3 de Julio]. 